\title{
REVUE
}

\section{L'EIMPLOI DES RÉCIPIENTS EN BOIS POUR LE TRANSPORT DU LAIT}

\author{
par G. GENIN
}

On a fréquemment songé à utiliser les récipients en bois pour le transport du lait et, dans certains cas, on a même envisagé l'emploi de petits tonnelets en bois pour le stockage et les livraisons de ce produit. Toutefois, certains inconvénients ayant résulté de l'utilisation de ces récipients, la question s'est posée de savoir si le chêne, par exemple, pouvait être utilisé tel quel pour la fabrication de ces tonnelets ou si au contraire il fallait protéger le bois par un revêtement approprié et, dans ce cas, lequel ?

La question a été récemment étudiée par un ingénieur allemand, le $\mathrm{D}^{\mathrm{r}} \mathrm{A}$. Herrmann, qui a publié le compte rendu de ses études dans la revue Farben Zeitung, et qui a montré qu'on pouvait parfaitement employer les récipients en bois à condition de les protéger par une peinture appropriée. Pour le choix de cette peinture, on a examiné, parmi de nombreux types de revêtement, ceux qui possédaient les qualités suivantes :

$1^{\circ}$ Une bonne résistance à l'acidité du lait.

$2^{\circ}$ Aucune influence sur le goût, l'odeur et l'aspect du lait.

$3^{\circ}$ Une résistance parfaite aux réactifs employés pour le nettoyage et la stérilisation des récipients.

C'est à la suite de plusieurs séries d'essais que l'on a pu mettre en évidence le revêtement le meilleur pour la protection du bois et nous décrirons aussi succinctement que possible les recherches effectuées dans ces différentes séries d'essai et les résultats auxquels elles ont conduit.

\section{PREIMIÈRE SÉRIE d'ESSAIS}

Au cours de cette première série d'essais, il a été examiné les points suivants :

$1^{0}$ Teneur en tanin des douves de chêne utilisées.

$2^{\circ}$ Teneur en humidité de ces douves avant et après essai. l'essai.

$3^{\circ}$ Conductibilité électrique du lait au début et à la fin de

$4^{\circ}$ Influence de l'acidité du lait sur la peinture protégeant le chêne.

$5^{\circ}$ Influence du nettoyage à l'eau chaude des douves sur la tenue de ces pièces.

$6^{\circ}$ Influence du récipient en bois sur la couleur, l'odeur et le goût du lait. 
Des essais bactériologiques étaient également effectués en collaboration avec le laboratoire bactériologique $d u \mathrm{D}^{\mathrm{r}} \mathrm{KUHL}$. Les recherches portèrent sur 60 douves de chêne d'une longueur de $450 \mathrm{~mm}$. polies sur leurs faces. On déterminait avant et après essai la teneur en tanin et en humidité de ces douves. Trois de ces douves étaient utilisées comme témoins, les 57 autres furent protégées soit par une peinture, soit par un produit d'imprégnation, et on utilisa à cet effet 19 produits remis par 13 firmes différentes.

Ces douves furent alors placées dans des bocaux partiellement remplis de lait écrémé dont on avait au préalable déterminé la conductibilité électrique. Ces bocaux étaient fermés par des couvercles en carton percés d'un trou pour le passage de la partie supérieure de la douve. L'espace libre compris entre la douve et les bords de l'orifice était obturé par un tampon d'ouate.

Après 10 jours de contact du lait et des douves, l'essai fut interrompu et on détermina la conductibilité électrique du lait, la teneur en humidité des douves, ainsi que les modifications apparentes subies par le lait. Les douves furent alors plongées pendant 10 minutes dans l'eau bouillante en vue de les stériliser, puis placées comme précédemment dans des bocaux remplis de lait frais dont on avait mesuré la conductivité électrique. Après 10 jours, les essais furent à nouveau interrompus et on procéda à un examen du lait et $\mathrm{du}$ bois.

Finalement les douves traitées par les 5 meilleurs produits examinés furent stérilisées et immergées à nouveau dans le récipient également stérilisé, rempli de lait écrémé stérilisé. Après 6 jours de contact, l'essai fut interrompu, on détermina l'humidité contenue dans les douves et on procéda également à des examens bactériologiques.

Les simples mesures de la teneur en humidité des douves donnèrent déjà des indications précieuses en accord avec l'essai mycologique et permirent d'éliminer rapidement un certain nombre de peintures qui s'étaient rapidement révélées insuffisantes (voir Tableau I).

D'autre part, la stérilisation effectuée par immersion dans l'eau, bouillante, car l'emploi de vapeur surchauffée n'était guère applicable en laboratoire, montra qu'un grand nombre des peintures essayées ne pouvaient supporter ce traitement. Il se produisait un changement de coloration et même, dans certains cas, une destruction visible de la pellicule. D'ailleurs, lorsque la peinture était détruite par ce traitement, il en résultait une augmentation sensible de la teneur en humidité de la douve, ce qu'on pouvait mettre facilement en évidence par une mesure électrique. 
TABLEAU I.

Résultats de la première série d'essais

\begin{tabular}{|c|c|c|c|c|}
\hline \multirow[b]{2}{*}{$\begin{array}{c}\text { Eprou- } \\
\text { vette } \\
\text { No }\end{array}$} & \multicolumn{3}{|c|}{$\begin{array}{c}\text { Teneur en humidité des } \\
\text { douves }\end{array}$} & \multirow[b]{2}{*}{ Observations } \\
\hline & $\begin{array}{r}\text { Avant } \\
\text { essai }\end{array}$ & \begin{tabular}{|c|} 
Après \\
10 jours \\
d'im- \\
mersion \\
\end{tabular} & $\mid \begin{array}{c}\text { Après } \\
20 \text { jours } \\
\text { d'im- } \\
\text { mersion }\end{array}$ & \\
\hline $1 \ldots \ldots$ & 11,0 & 14,6 & $>22,0$ & Faibles amas de moisissures. \\
\hline $2 \ldots \ldots$ & 10,8 & $>22,0$ & $>22,0$ & $\begin{array}{l}\text { Changement de couleur. Faible formation } \\
\text { de moisissures. }\end{array}$ \\
\hline $3 \ldots \ldots$ & 11,0 & $>22,0$ & $>22,0$ & $\begin{array}{l}\text { Faible formation de moisissures. Lait à } \\
\text { odeur de bois. }\end{array}$ \\
\hline $4 \ldots \ldots$ & 10,7 & 14,8 & 17,7 & Faible formation de moisissures. \\
\hline $5 \ldots \ldots$ & 10,8 & 11,6 & 19,7 & \\
\hline $6 \ldots \ldots$ & 10,8 & 12,2 & 17,2 & \\
\hline $7 \ldots \ldots$ & 11,6 & 14,6 & $>22,0$ & Faible formation de moisissures. \\
\hline $8 \ldots \ldots$ & 11,0 & 14,0 & $>22,0$ & \\
\hline $9 \ldots \ldots$ & 11,0 & 11,2 & 13,0 & \\
\hline $10 \ldots \ldots$ & 10,8 & 13,3 & 18,5 & \\
\hline $11 \ldots \ldots$ & 10,9 & 11,0 & 18,0 & ' \\
\hline $12 \ldots \ldots$ & 10,5 & 11,0 & $>22,0$ & \\
\hline $13 \ldots \ldots$ & 11,0 & 17,3 & $>22,0$ & $\begin{array}{l}\text { Faible formation de moisissures. Peinture } \\
\text { devenue plus claire. }\end{array}$ \\
\hline $14 \ldots \ldots$ & 11,2 & 13,2 & 19,0 & (1) \\
\hline $15 \ldots \ldots$ & 10,5 & 10,9 & 11,3 & \\
\hline $16 \ldots \ldots$ & 11,1 & 15,3 & $>22,0$ & Peinture devenue plus foncée. \\
\hline $17 \ldots \ldots$ & 10,8 & $>22,0$ & $>22,0$ & $\begin{array}{l}\text { Forte formation de moisissures. Lait à } \\
\text { odeur de bois. }\end{array}$ \\
\hline $18 \ldots \ldots$ & 11,2 & 17,0 & $>22,0$ & '. \\
\hline $19 \ldots \ldots$ & 10,9 & 15,5 & 20,3 & . \\
\hline $20(1) \ldots$ & 10,5 & $>22,0$ & $>22,0$ & $\begin{array}{l}\text { Forte formation de moisissures. Lait à } \\
\text { odeur de bois. }\end{array}$ \\
\hline
\end{tabular}

(1) Echantillon témoin non recouvert de peinture.

Par contre, la détermination de la conductivité électrique du lait n'a pas permis de mettre en évidence la supériorité d'une peinture ou d'une autre et de même on a observé que l'acidité du lait a une influence très faible sur la teneur des peintures.

On a constaté l'existence d'une zone sensible à la surface de séparation liquide et air où se produit un fort développement de penicillium vert. Les douves bien protégées par une couche de peinture parfaite étaient exemptes de ce phénomène qui au contraire était abondant dans les douves mal protégées.

De l'ensemble des échantillons de peinture examinés au cours 
de cette première série d'essais, 5 s'étaient révélés intéressants, le lait placé au contact des douves protégées par ces peintures, qu'il soit frais ou écrémé, n'ayant acquis aucun goût, aucune coloration, ni aucune odeur particuliers.

\section{DEUXIËME SÉRIE D'ESSAIS}

Nous ne dirons que quelques mots de cette seconde série d'essais dont le principal but était de reproduire et de confirmer les observations faites au cours de la première série. Les recherches portèrent d'une part sur certaines peintures dont la qualité avait été mise en évidence au cours de la première série d'études et d'autre part sur quelques peintures nouvelles. L'examen bactériologique fut supprimé, mais, par contre, on apporta beaucoup d'importance à la question de la détermination de la teneur en humidité des douves et de leur teneur en tanin. Les conditions de l'essai au point de vue de la durée d'immersion dans l'eau et de la stérilisation étaient les mêmes que précédemment.

On a ainsi pu mettre en évidence 5 types de peintures différentes permettant d'obtenir des douves dont la teneur en humidité après 20 jours d'immersion dans l'eau n'était pas supérieure à $17 \%$. Ce sont ces peintures qui furent alors examinées d'une façon plus approfondie dans la troisième série d'essais.

\section{TROISIÈME SÉRIE D'ESSAIS}

Ces essais ont eu un caractère plus pratique, en ce sens qu'au lieu de porter sur des douves peintes, ils furent effectués sur de petits tonnelets en bois qui servirent effectivement au transport et à l'expédition du lait. L'examen des récipients après transport permit de montrer la qualité de certaines peintures et les observations faites furent confirmées par des mesures de teneur en humidité du bois et par un examen bactériologique du lait.

En réalité, les tonnelets utilisés s'écartaient légèrement de ceux qu'on emploie couramment pour le transport des liquides par le fait que leur couvercle était entièrement mobile afin de faciliter le nettoyage et la stérilisation, le joint étant assuré par un anneau de caoutchouc. La contenance des récipients était d'environ 20 litres. Huit peintures reconnues favorables au cours des essais extérieurs furent étudiées à nouveau et les essais portèrent également sur un tonnelet en bois non peint, ainsi que sur des récipients métalliques.

Tout d'abord ces tonnelets furent remplis d'eau renouvelée tous les 3 jours. Après 4 semaines de contact avec l'eau, on détermina la teneur en humidité du bois, puis les récipients furent envoyés à une importante laiterie qui procéda à leur stérilisation avant d'introduire le lait écrémé. Cette stérilisation s'effectuait en introduisant pendant 3 à 4 secondes de la vapeur dans le tonnelet, on constatait 
l'apparition d'une odeur de peinture plus ou moins intense qui disparaissait d'ailleurs presque entièrement.

Après remplissage avec du lait écrémé, pasteurisé et froid, les récipients furent remis aux chemins de fer pour un voyage aller et retour d'une durée d'environ 3 jours. Au retour, les récipients furent examinés au point de vue de la teneur en humidité, de l'état de la peinture et des qualités dégustatives du lait. Les récipients furent à nouveau stérilisés à la vapeur et abandonnés dans une salle dont la température était maintenue à environ $9^{\circ}$. Après environ 1 mois de repos, les récipients furent à nouveau stérilisés à la vapeur, remplis de lait écrémé et abandonnés dans une pièce dont la température était maintenue à $27^{\circ}$ et dont l'atmosphère avait une teneur en humidité de $45 \%$. Au bout de ce temps, le contenu des récipients fut examiné, ainsi que l'état de la peinture.

Le simple contact de l'eau et des récipients n'a permis de faire avcune observation. Après l'essai de transport, on a mesuré la teneur en humidité des différents bois et on a obtenu des valeurs qui sont reportées dans le tableau ci-dessous :

TABLEAU II.

\begin{tabular}{|c|c|c|}
\hline \multirow{2}{*}{ Échantillons No } & \multicolumn{2}{|c|}{ Teneur en humdité du bois } \\
\hline & Avant transport & Après transport \\
\hline $1 \ldots \ldots \ldots \ldots \ldots \ldots$ & 8,9 & 14,2 \\
\hline $2 \ldots \ldots \ldots \ldots \ldots \ldots \ldots$ & 10,7 & 15,2 \\
\hline $3 \ldots \ldots \ldots \ldots \ldots \ldots \ldots \ldots$ & 10,4 & 13,4 \\
\hline $4 \ldots \ldots \ldots \ldots \ldots \ldots \ldots \ldots$ & 10,3 & 14,0 \\
\hline $5 \ldots \ldots \ldots \ldots \ldots \ldots \ldots$ & 8,5 & 11,6 \\
\hline $6 \ldots \ldots \ldots \ldots \ldots \ldots \ldots$ & 10,9 & 14,9 \\
\hline $7 \ldots \ldots \ldots \ldots \ldots \ldots \ldots \ldots \ldots \ldots$ & 10,9 & 14,3 \\
\hline $8 \ldots \ldots \ldots \ldots \ldots \ldots \ldots \ldots \ldots$ & 2,9 & 14,7 \\
\hline 9 (sans peinture) $\ldots \ldots \ldots \ldots$ & 14,6 & 17,7 \\
\hline
\end{tabular}

On notera la faible teneur en humidité des échantillons recouverts par une couche de peinture par rapport aux échantillons non traités.

$\mathrm{Au}$ cours du stockage des récipients dans une pièce tempérée, on a pu constater à la fin de la période de stockage qu'une odeur de moisi se manifestait dans les récipients, odeur d'autant plus prononcée que le récipient n'avait pas été traité, mais qui disparaissait par la suite. Si on procédait à l'observation de l'état du lait, aucune modification anormale n'était mise en évidence dans le lait contenu dans des tonnelets en bois, alors qu'on trouvait des faibles traces de métaux dans le lait contenu dans des récipients métalliques.

En résumé, les expériences ont montré que les tonnelets en chêne 
protégés par un revêtement de peinture peuvent être utilisés pour le transport du lait, surtout si le mode de construction du tonnelet permet un enlèvement complet du couvercle et par conséquent un nettoyage efficace de l'intérieur du récipient.

\section{BIBLIOGRAPHIE ANALYTIQUE}

\section{LES LIVRES}

\section{Hunziker (O. F.). - Condensed milk and milk powder (Lait} condensé et poudre de lait). Publié par l'auteur. La Grange, Illinois (Etats-Unis d'Amérique), 1935, un livre relié toile de 661 pages, a vee 148 figures et 63 tableaux.

Nos lecteurs connaissent certainement déjà ce livre qui est classique, au même titre que les autres ouvrages de M. Hunziker. En voici la $5^{\ominus}$ édition, revue et augmentée, la $1^{\text {re }}$ datant, si nos renseignements sont exacts, de 1914 , Euvre d'un praticien, doublé d'un savant, le travail de M. H. est complet et entièrement à jour. Il se compose de sept parties : Généralités sur le lait condensé ; Fabrication du lait condensé sucré ; Fabrication du lait condensé non sucré ; Vente des laits condensés ; leurs compositions et leurs propriétés ; Défauts des laits condensés ; leurs causes ; comment les éviter ; Fabrication de la poudre de lait ; Essais, analyses et standards légaux.

Tout est à lire dans cet important volume. Les étudiants et les hommes de science y trouveront une documentation claire, détaillée et à jour ; les indus triels, des renseignements complets sur la fabrication et les divers emplois des laits condensés, de la poudre de lait et du sucre de lait, ainsi que sur d'autres produits tels que le babeurre et le sérum en poudre, le lait malté, le babeurre demi-solide, le lait écrémé acide concentré, et la crème douce stérilisée.

\section{Wolf.}

Davies (W. L.). - The Chemistry of Milk (La chimie du lait). Un livre relié toile de 522 pages. Editeurs : Chapman and Hall Ltd, 11 Henrietta Street, London, W. C. 2. 1936. Prix net : 25 shillings.

L'ouvrage de M. Davies porte le no 10 dans une série de monographies publiée sous la direction de M. E. How ARD TRIPP. Il présente l'état actuel de la chimie du lait d'après les travaux les plus récents. Le mérite de M. D. n'est pas mince. Il a compulsé un très grand nombre de livres, lu avec attention tous les périodiques traitant des questions laitières, pour ne retenir, suivant l'expression de M. Howard TrIPp, que ce qui lui a paru avoir une valeur soit actuelle, soit potentielle. On se rendra compte du travail fourni quand on saura que 1.400 références sont incluses dans le texte.

Le résultat est excellent. Le livre de M. D. dit ce qu'il faut dire, dans une langue simple et élégante. Il est divisé en cinq parties : Composition du lait; Constituants du lait ; Physico-chimie du lait ; Chimie des traitements du 